\title{
Analysis of by high-throughput sequencing: Helicobacter pylori infection and salivary microbiome
}

\author{
Yingjie Ji, Xiao Liang and Hong Lu*
}

\begin{abstract}
s
Background: There have been reports of Helicobacter pylori (H. pylori) in the oral cavity and it has been suggested that the oral cavity may be a reservoir for $\mathrm{H}$. pylori reflux from the stomach. High-throughput sequencing was used to assess the structure and composition of oral microbiota communities in individuals with or without confirmed $H$. pylori infection.
\end{abstract}

Methods: Saliva samples were obtained from 34 H. pylori infected and 24 H. pylori uninfected subjects. Bacterial genomic DNA was extracted and examined by sequencing by amplification of the 165 rDNA V3-V4 hypervariable regions followed by bioinformatics analysis. Saliva sampling was repeated from 22 of the $34 \mathrm{H}$. pylori infected subjects 2 months after $H$. pylori eradication.

Results: High-quality sequences $(2,812,659)$ clustered into 95,812 operational taxonomic units (OTUs; $97 \%$ identity). H. pylori was detected in the oral cavity in infected (12/34), uninfected (11/24) and eradicated (15/22) subjects by technique of high-throughput sequencing, occupying $0.0139 \%$ of the total sequences. Alpha diversity of $\mathrm{H}$. pylori infected subjects was similar to that of uninfected subjects (Shannon: 1417.58 vs. 1393.60, $p>0.05$, ACE: 1491.22 vs. 1465.97, $p>0.05$, Chao 1: 1417.58 vs. 1393.60, $p>0.05$, $t$-test). Eradication treatment decreased salivary bacterial diversity (Shannon, $p=0.015, \mathrm{ACE}, p=0.003$, Chao $1, p=0.002$, $t$-test). Beta diversity analysis based on unweighted UniFrac distances showed that the salivary microbial community structure differed between $H$. pylori infected and uninfected subjects (PERMANOVAR, pseudo-F: 1.49, $p=0.033$ ), as well as before and after H. pylori eradication (PERMANOVAR, pseudo-F: 3.34, $p=0.001$ ). Using LEfSe analysis, 16 differentially abundant genera were defined between infected and uninfected subjects, 12 of which had a further alteration after successful eradication.

Conclusions: Our study using high-throughput sequencing showed that $\mathrm{H}$. pylori was present commonly in the oral cavity with no clear relation to $\mathrm{H}$. pylori infection of the stomach. Both $\mathrm{H}$. pylori infection and eradication therapy caused alterations in community and structure of the oral microbiota.

Trial registration: clinicaltrials.gov, NCT03730766. Registered 2 Nov 2018 - Retrospectively registered, https:/clinicaltrials. gov/ct2/show/ NCT03730766.

Keywords: Helicobacter pylori, Salivary microbiota, High-throughput sequencing, 165 rDNA

\footnotetext{
* Correspondence: hlu@sjtu.edu.cn

Division of Gastroenterology and Hepatology, Key Laboratory of Gastroenterology and Hepatology, Ministry of Health, Renji Hospital, School of Medicine, Shanghai Jiao Tong University, Shanghai, China
} 


\section{Background}

H. pylori is a Gram-negative bacterium that colonizes the human gastric epithelium. H. pylori infection is characterized by mucosal inflammation (gastritis) and may result in peptic ulcer disease or gastric adenocarcinoma [1]. H. pylori is transmitted between humans by a variety of routes including gastro-oral and fecal-oral mechanisms that include contaminated water and food. It has also been postulated that oral cavity may play a role in H. pylori transmission and possibly act as a reservoir [2]. For example, $H$. pylori has been detected in the oral cavity using the polymerase chain reaction (PCR). H. pylori has also been successfully cultured from saliva from individuals with positive results of both saliva $H$. pylori antigen test and $H$. pylori flagella test [3].

The oral cavity is one of the most complex and largest microbial habitats that harbors hundreds of different bacteria which play important roles in maintaining oral homeostasis and influencing the development of both oral and systematic diseases [4]. Many factors in the oral environment including intraoral $\mathrm{pH}$ and salivary iron concentration as well as expression of serum malondialdehyde and lipid profile have been reported to have significant relationships with oral microbial communities $[5,6]$. Several recent studies detected the efficacy of antibiotics or drug composed of herbal on oral microbiol communities [7, 8]. However, there was few reports about $H$. pylori and its relationship with the microbial community structure in human saliva.

Currently, most oral bacteria species cannot be easily cultivated in vitro using traditional cultivation methods requiring the use of molecular biological techniques, such as checkerboard hybridization, microarray chips, and the quantitative real-time PCR to identify and classify the currently uncultivable bacteria [9]. However, many low-abundance bacteria species still cannot be detected using these approaches which impede the comprehensive and in-depth understanding of oral bacteria diversity. In this study, we used amplicon sequencing of $16 \mathrm{~S}$ rDNA V3-V4 hypervariable regions to define the bacterial composition, abundance, and structure of salivary microbiome in people with and without active $H$. pylori infections. In addition, we also characterized the salivary biodiversity of a subgroup of subjects before and after the $H$. pylori eradication. As $H$. pylori passes through saliva, it is important to detect its expression in oral cavity as well as the efficacy of its infection and eradication on the oral microbiol communities.

\section{Methods}

\section{Subjects and sample collections}

The samples were collected in Renji Hospital of Shanghai Jiao Tong University, China, from August to November in 2018. A total of 58 subjects were recruited, including 34 subjects with $H$. pylori infection and 24 uninfected subjects. We first conducted a cross-sectional study of the salivary microbiota of $34 \mathrm{H}$. pylori infected and 24 uninfected subjects. A prospective study was then performed in a subgroup of 22 subjects with $H$. pylori infection who underwent salivary analysis both before and after successful $H$. pylori eradication.

All subjects received both endoscopy and ${ }^{13} \mathrm{C}$ urea breath test $\left({ }^{13} \mathrm{C}\right.$-UBT $)$ before enrollment. The $H$. pylori infection status was confirmed by positive rapid urease test (RUT), histology and ${ }^{13} \mathrm{C}$-UBT. Absence of infection was defined as negative results for all tests (i.e., RUT, histology and ${ }^{13} \mathrm{C}$-UBT). H. pylori infected subjects received eradiation therapy consisting of esomeprazole 20 mg b.i.d., bismuth potassium citrate $600 \mathrm{mg}$ b.i.d, amoxicillin $1000 \mathrm{mg}$ t.i.d., and metronidazole $400 \mathrm{mg}$ t.i.d. for 14 days. $H$. pylori eradication was confirmed by ${ }^{13} \mathrm{C}$-UBT at least 6 weeks after the end of treatment. Saliva samples were collected from 22 subjects both before and 2 months following successful $H$. pylori eradication. Subjects were characterized into four groups. $H$. pylori uninfected group (uninfected) $(n=24), H$. pylori infected group (infected) $(n=34)$, and Pre-eradicated $H$. pylori infected group (pre-eradicated) $(n=22)$ and a successful eradicated group (eradicated) $(n=22)$. Inclusion criteria of subjects were: age of 20-65 years old male or female, with good oral hygiene (including brushing teeth twice a day for three minutes each) and with no bad eating habits [10]. Exclusion criteria included: 1) the presence of dental carious or any untreated cavitated carious lesions and oral abscesses, 2) periodontal disease or periodontal pockets $\geq 4 \mathrm{~mm}, 3$ ) the use of antibiotics or proton pump inhibitors (PPIs) within 2 months before the study, 4) previous diagnosis of a serious systemic diseases (such as diabetes, hypertension or cardiopathy) or any diseases affecting oral health (such as Sjogren's syndrome or any disease characterized by xerostomia), 5) pregnancy of breastfeeding, 6) specific eating habits or habits of eating specific food such as garlic or pepper and 7) smoking or alcohol drinking. The periodontal examinations were conducted by the same professional dentist in Renji Hospital of Shanghai Jiao Tong University, China, using the same diagnostic criteria. The detailed clinical parameters of the 58 subjects are shown in Additional file 1:Table S1.

\section{Salivary sampling}

Sampling was performed in the morning $2 \mathrm{~h}$ after eating. Saliva samples were collected from each subject according to the Manual of Procedures for Human Microbiome Project (http://hmpdacc.org/resources/tools protocols.php), with minor modifications. Approximately 3-4 $\mathrm{ml}$ of non-stimulated saliva was collected in two sterile, labeled $2 \mathrm{~mL}$ Eppendorf tubes, which were 
immediately placed on ice. Within $3 \mathrm{~h}$ of collection, samples were transported on ice and stored at $-80^{\circ} \mathrm{C}$ until use.

\section{DNA extraction and sequencing}

DNA was extracted from the saliva samples using the E.Z.N.A. ${ }^{\circ}$ Soil DNA Kit (OMEGA, USA), following the manufacturer's instructions, and stored at $-20^{\circ} \mathrm{C}$ prior to further analysis. PCR amplification of the bacterial 16S rDNA hypervariable V3-V4 region was performed using the forward primer 338F (5'-ACTCCTACGGGA GGCAGCA-3') and the reverse primer 806R (5'GGACTACHVGGGTWTCTAAT-3'). Sample-specific 7-bp barcodes were incorporated into the primers for multiplex sequencing. Details of the barcodes are shown in Additional file 2: Table S2. PCR amplification were performed on an ABI 2720 instrument (ABI, USA) with an initial denaturation at $98^{\circ} \mathrm{C}$ for $2 \mathrm{~min}$, followed by 25 cycles of denaturation $\left(15 \mathrm{~s}\right.$ at $98^{\circ} \mathrm{C}$ ), annealing (30s at $55^{\circ} \mathrm{C}$ ), extension $\left(30 \mathrm{~s}\right.$ at $\left.72^{\circ} \mathrm{C}\right)$, and ended with a final extension $\left(5 \mathrm{~min}\right.$ at $\left.72^{\circ} \mathrm{C}\right)$. A negative control was set during the process of PCR amplification to eliminate reagent contamination. PCR amplicons were purified with Agencourt AMPure Beads (Beckman Coulter, Indianapolis, IN) and quantified using the Quant-iT PicoGreen dsDNA Assay Kit (Invitrogen, Carlsbad, CA, USA). Equimolar concentrations of purified amplicons were pooled in equal amounts. Subsequently, the paired-end $2 \times 300$ bp sequencing plus $20 \%$ PhiX control DNA was performed on the Illumina MiSeq platform with MiSeq Reagent Kit v3 (Illumina, USA), following the vendor's standard protocols.

\section{Sequence analysis}

The Quantitative Insights Into Microbial Ecology (QIIME) pipeline was employed to process the sequencing data. Raw sequences were filtered to obtain highquality sequences according to QIIME [11]. The lowquality sequences were filtered through following criteria: sequences that had a length of $<200 \mathrm{bp}$, sequences that had average Phred scores of $<25$, sequences that contained ambiguous bases or homopolymeric stretches, and sequences that contained mononucleotide repeats of $>8 \mathrm{bp}$. The high-quality sequences were clustered into operational taxonomic units (OTUs) at 97\% sequence identity by UCLUST [12]. The representative sequences selected from each OTU were classified taxonomically by BLAST searching against the Human Oral Microbiome Database (HOMD). OTU taxonomic classification was conducted by BLAST searching the representative sequences set against the HOMD using the best hit, with a BLAST e-value $<1 \mathrm{e}-5$. HOMD provides a detailed record of the type, metabolism, and pathogenicity of oral bacteria [13]. Then, an OTU table was further generated to record the OTU abundance of each sample and the taxonomic classification of these OTUs. Finally, to minimize the difference of sequencing depth across samples, the OTU table was modified by removing OTUs containing less than $0.001 \%$ of total sequences across all samples for further analysis [14].

\section{Bioinformatics and statistical analysis}

Sequence data analyses were mainly performed using QIIME (version 1.8.0) and $\mathrm{R}$ packages (version 3.2.4). The alpha diversity analysis including Chao 1 richness estimator, Abundance-based Coverage Estimator (ACE) metric, Shannon diversity index, and Simpson index, were calculated at 97\% identity using the QIIME [15]. Ranked abundance curves were generated to compare both the richness and evenness of OTUs among samples. The beta diversity analysis including Nonmetric Multidimensional Scaling (NMDS), and unweighted UniFrac distances based principal coordinate analysis ( $\mathrm{PCoA}$ ), were performed using the $\mathrm{R}$ package to evaluate the similarity among various bacterial communities [16]. The significance of differentiation of microbiota structure among groups was assessed by Adonis test [17]. The taxonomy compositions and abundances were visualized by MEGAN (version 6.6.7) software [18]. Linear discriminant analysis effect size (LEfSe) was used to compare the bacterial community structures between the samples from the patients with and without $H$. pylori infection, as well as before and after the eradication regimen, using the online Galaxy workflow framework (http://huttenhower.sph.harvard.edu/galaxy/) [19].

\section{Results}

\section{Global sequencing data}

The saliva samples were obtained from $34 \mathrm{H}$. pylori infected, 24 uninfected and 22 in 34 subjects 2 months after successful eradication therapy. A total of 2,812,659 high-quality sequences (representing $79 \%$ of the total sequences) were acquired from the 80 saliva samples, with an average of 35,158 sequences per sample (ranging from 19,210 to 44,310; Additional file 3: Table S3). The average sequence length was $445 \mathrm{bp}$, with the maximum length being $548 \mathrm{bp}$ and the shortest length being $136 \mathrm{bp}$ (Additional file 4: Figure S1). Clustering of all highquality sequences at $97 \%$ identity by UCLUST resulted in 70,489 OTUs with an average of 4222 OTUs (ranging from 2170 to 5669), which were BLAST-searched against the HOMD for taxonomic assignments. After removing the low-credibility OTUs (together contributing only $6.7 \%$ of all sequences) and subsampling each sample to an equal sequencing depth of 19,210 reads per sample, a modified OTU table was obtained consisting of 95,812 OTUs with an average of 1198 OTUs per sample (ranging from 697 to 1584; Additional file 5: Table S4). 


\section{Bacterial abundance and distribution}

The bacterial distribution was characterized in terms of the relative taxonomic abundances. A total of 11 phyla, 21 classes, 36 orders, 68 families, 138 genera and 440 species were detected in the saliva samples. The taxonomic distributions of the predominant bacteria (relative abundance $>1 \%$ of the total sequences) in subjects with and without $H$. pylori at different levels were shown in Fig. 1. The 6 most abundant phyla were Proteobacteria (40.1\% of the total sequences), Firmicutes (31.6\%), Bacteroidetes (13.0\%), Actinobacteria (7.4\%), Fusobacteria (6.1\%), and TM7 (1.0\%), together accounting for $99.2 \%$ of the total sequences. At genus level, saliva microbiota was dominated by Neisseria, Streptococcus, Haemophilus, Veillonella, and Prevotella, with average relative abundances of 20.2, 16.5, 10.5, 8.0, and 8.0\%, respectively. Fusobacteria was observed higher abundance in eradicated group on phyla level. Leptotrichia, Campylobacter and Pseucomonas expressed higher in eradicated group on genus level, while Alloprevotella and Aggregatibacter were observed higher abundance in uninfected group. The compositions in taxa of the microbial communities according to the tested sample groupings are provided in Additional file 6: Figure S2.

\section{Bacterial diversity analysis}

The salivary microbiota richness, measured by numbers of observed OTUs, was similar in uninfected subjects and infected subjects (Additional file 7: Figure S3A). The alpha diversity indices of Chao1, ACE, Shannon, Inverse Simpson, Good's coverage, and Simpson's evenness are shown in Table 1. The Shannon diversity index was higher in uninfected subjects than in infected subjects, but there was no significant difference between groups by $t$-test $(1417.58$ vs. $1393.60, p>0.05)$. Besides, the ACE richness index (1491.22 vs. 1465.97, $p>0.05)$, Chao 1 richness estimator (1417.58 vs. $1393.60, p>0.05)$, and the Inverse Simpson diversity index (1.02 vs. $1.02, p>0.05)$ was

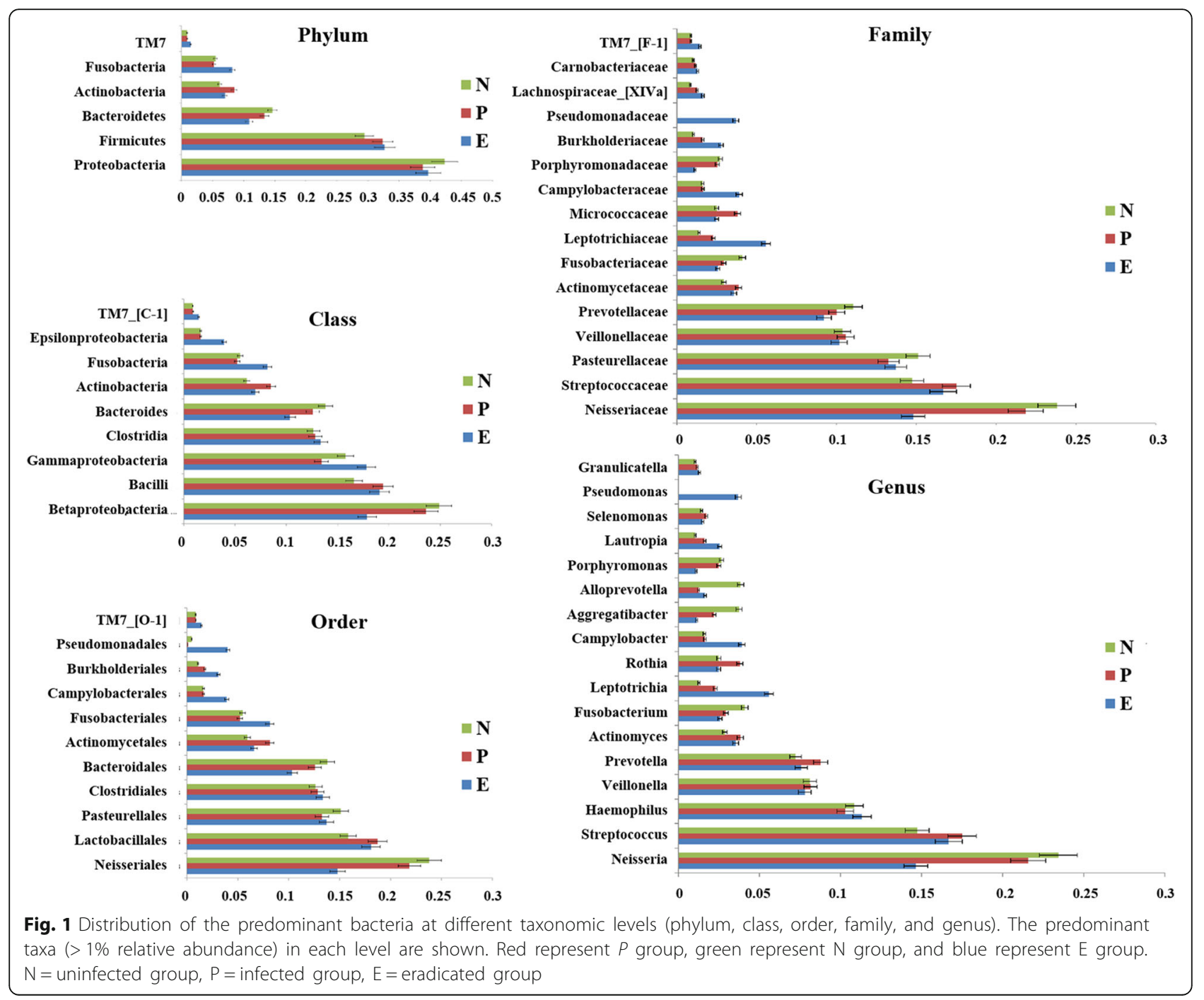


Table 1 Alpha diversity indices for saliva bacteria in each group at 97\% identity

\begin{tabular}{|c|c|c|c|c|c|c|c|c|c|c|c|c|}
\hline \multirow[t]{2}{*}{ Group } & \multicolumn{2}{|l|}{ Chao1 } & \multicolumn{2}{|l|}{ ACE } & \multicolumn{2}{|c|}{ Shannon } & \multicolumn{2}{|c|}{ Inverse Simpson } & \multicolumn{2}{|c|}{ Coverage } & \multicolumn{2}{|c|}{ Simpsoneven } \\
\hline & Mean & SD & Mean & SD & Mean & SD & Mean & SD & Mean & SD & Mean & SD \\
\hline $\mathbf{N}$ & 1417.58 & 195.47 & 1491.22 & 210.41 & 7.94 & 0.36 & 1.02 & 0.01 & 0.98 & 0.01 & 0.05 & 0.02 \\
\hline $\mathbf{P}$ & 1393.60 & 182.57 & 1465.97 & 196.06 & 7.89 & 0.42 & 1.02 & 0.01 & 0.98 & 0.00 & 0.05 & 0.01 \\
\hline
\end{tabular}

SD Standard Deviation. $N$ uninfected group, $P$ infected group

No statistically significant difference was observed in all index among N group and P group ( $p>0.05$, Student's t-test)

also higher in uninfected subjects, with no significant difference, indicating the similar bacterial diversity of $H$. pylori uninfected saliva compared to the infected subjects. Good's coverage estimator for each group was over $98 \%$, indicating that the current sequencing depth was sufficient to saturate the bacterial diversity of saliva. In addition, Simpson's evenness index indicated that the bacterial-community distribution in two groups was uneven, which was also observed in the rank-abundance curve (Additional file 8: Figure S4).

\section{Bacterial community structures}

To gain insights into the similarities in the bacterial community structures among uninfected and infected subjects, PCoA of beta diversity analysis was performed based on the unweighted UniFrac distances, which demonstrated different community structures among two groups (PERMANOVAR, pseudo-F: $1.49, p=0.033$ ). As shown in Fig. 2a, the overall microbial composition of infected subjects deviated from that of uninfected subjects. Furthermore, the results of NMDS based on the genus level classification exhibited clear segregations in community structures among groups (Additional file 9: Figure S5A).

\section{Differential microbiota compositions}

There were significant differences in the community compositions among two groups. As shown in Fig. 3, a cladogram representation of significantly different taxa among groups was performed by LEfSe. The microbial composition was significantly different at the genus level, with 16 significantly different genera among the two groups. Acinetobacter, Ralstonia, Leptothrix, Sphingomonas, Ochrobactrum, Afipia, Leptotrichia, Oribacterium, and Moryella exhibited a relatively higher abundance in infected subjects, and can be considered $H$. pylorienriched genera. Alloprevotella, Aggregatibacter, Klebsiella, Leptotrichlaceae_G_1_, Fusobacterium, Parvimonas, and Peptococcus were relatively more abundant in uninfected subjects, which could be considered to be decreased in the infected group. These higher or lower expressed genera in infected subjects can be considered as $H$. pylori-associated genera (LAD $>2, p<0.05$ ).

\section{Eradication therapy for $H$. pylori partially changed salivary microbiota}

To determine the effects of $H$. pylori eradication therapy on salivary microbial composition, saliva samples from a
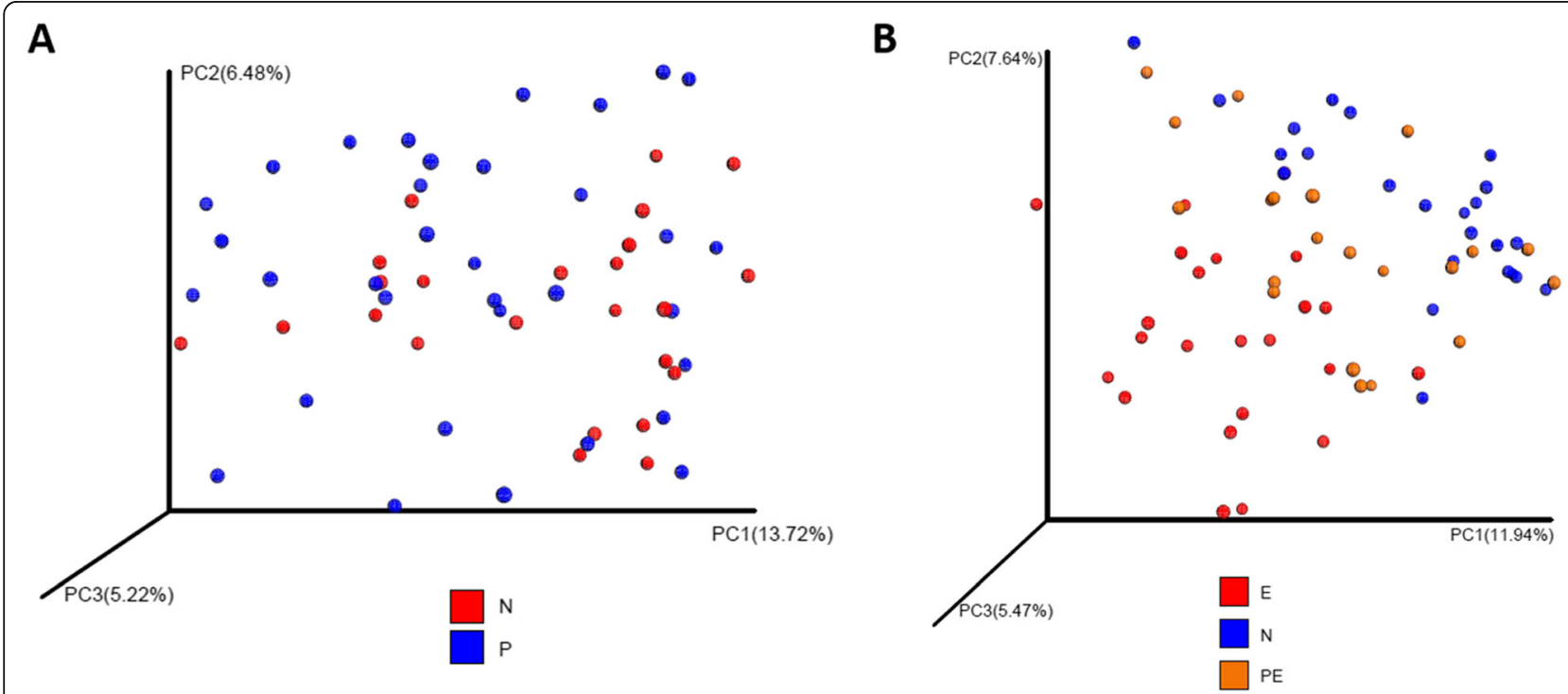

Fig. 2 Principal coordinate analysis (PCOA) of unweighted UniFrac analysis. a. PCoA analysis demonstrated that subjects of $P$ group were significantly different from $N$ group (PERMANOVAR, pseudo-F: 1.49, $p=0.033$ ). $N=$ uninfected group, $P=$ infected group. b. PCoA analysis showed that the overall microbial composition showed significant difference between PE and $\mathrm{E}$ group (PERMANOVAR, pseudo-F: $3.34, p=0.001)$. $E=$ eradicated group, $\mathrm{N}=$ uninfected group, $\mathrm{PE}=$ pre-eradicated group 


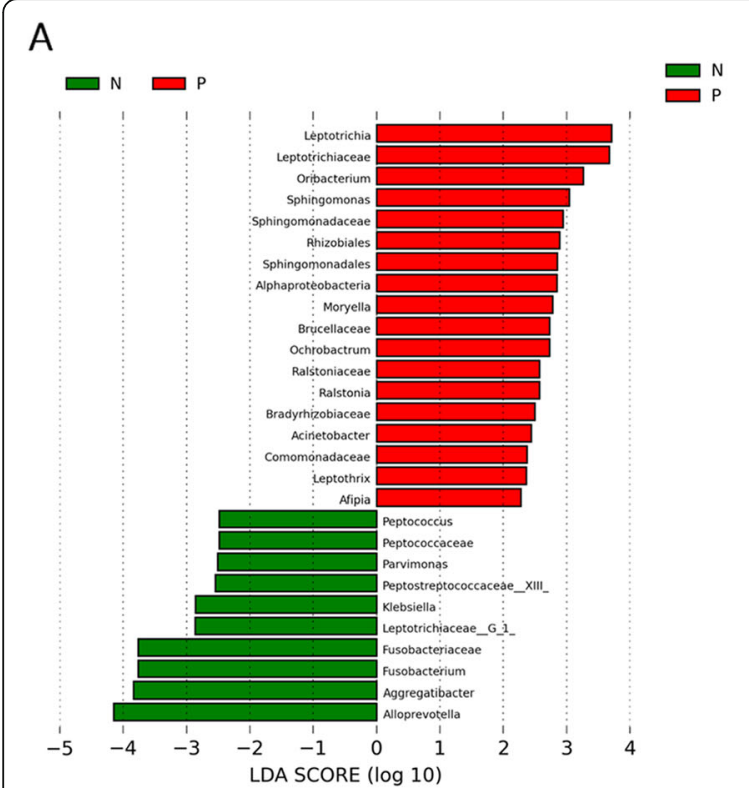

B
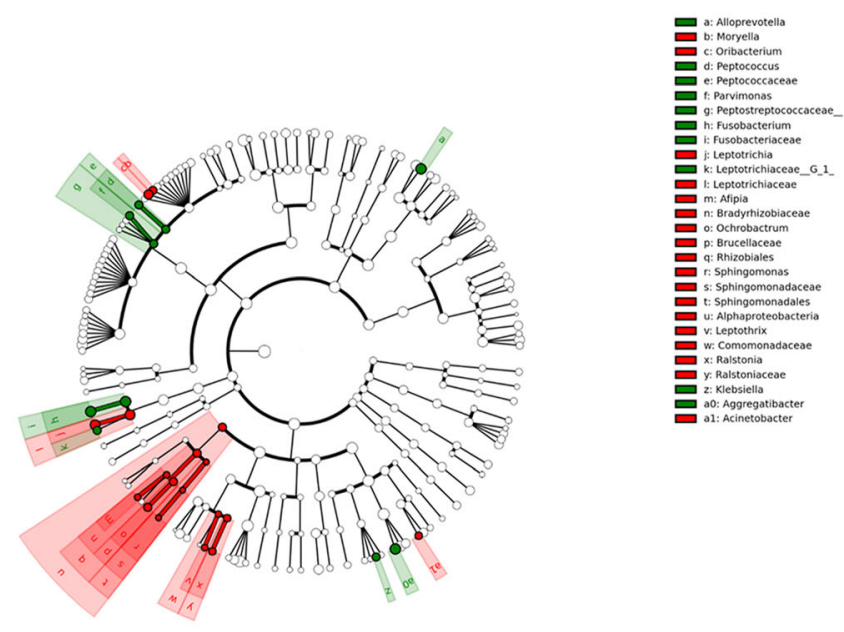

Fig. 3 Comparison of microbial variations at the genus level, using the LEfSe online tool. a. Histogram of the LDA scores for differentially abundant features among groups. The threshold on the logarithmic LDA score for discriminative features was set to 2.0. $N=$ uninfected group, $\mathrm{P}=$ infected group. $\mathbf{b}$. Cladogram for taxonomic representation of significantly differences among groups. Differences are represented in the color of the most abundant taxa (red indicating $P$ group, green indicating $N$ group, and white indicating non-significant). $N=$ uninfected group, $P=$ infected group

subgroup of $H$. pylori infected subjects $(n=22)$, were collected before (pre-eradicated group) and 2 months after treatment; saliva collected after successful eradication were classified into the eradicated group. The within-individual diversity in the samples from eradicated group was lower than pre-eradicated group (Additional file 7: Figure S3B). The Shannon diversity index, ACE richness index, and Chao 1 richness estimator were higher in pre-eradicated subjects than in eradicated subjects, with a significant difference between groups by $t$-test (Shannon $p=0.015$, ACE $p=0.003$, Chao $1 p=0.002$ ), indicating significant alteration in the within-individual diversity in samples from eradicated subjects compared to their baseline samples (Table 2).

The beta diversity using unweighted UniFrac showed significant differences in the overall microbial composition between pre-eradicated and eradicated groups (PERMANOVAR, pseudo-F: 3.34, $p=0.001$ ) (Fig. 2b). The NMDS also exhibited clear segregations in community structures among groups (Additional file 9:Figure S5B).
The relative difference of $H$. pylori-associated taxa was compared before and after eradication by LEFse analysis (Additional file 10:Figure S6). Among the H. pylorienriched genera, Ralstonia, Leptotrichia, Sphingomonas, Leptothrix, Oribacterium, and Acinetobacter increased after the eradication, while Ochrobactrum decreased after the successful eradication $(p<0.05$, paired Wilcoxon rank-sum test). Of the genera that decreased in infected subjects, Alloprevotella, Aggregatibacter, Leptotrichlaceae_G_1_, Parvimonas, and Fusobacterium decreased after the eradication $(p<0.05$, paired Wilcoxon rank-sum test). Besides, we found that at phyla level, Fusobacteria increased after $H$. pylori eradication.

\section{H. pylori in the oral cavity}

H. pylori was detected in 38 out of 80 saliva samples, regardless of the $H$. pylori status in stomach, occupying $0.0139 \%$ of all the total sequences. (Fig. 4). 12 of the 34 subjects in infected subjects (35.3\%), 11 of 24 subjects in the uninfected group (45.8\%), and 15 of 22 subjects in the eradicated group (68.2\%) were found to possess $H$.

Table 2 Alpha diversity indices for saliva bacteria in each group at 97\% identity

\begin{tabular}{|c|c|c|c|c|c|c|c|c|c|c|c|c|}
\hline \multirow[t]{2}{*}{ Group } & \multicolumn{2}{|l|}{ Chao1 } & \multicolumn{2}{|l|}{$\mathrm{ACE}$} & \multicolumn{2}{|c|}{ Shannon } & \multicolumn{2}{|c|}{ Inverse Simpson } & \multicolumn{2}{|c|}{ Coverage } & \multicolumn{2}{|c|}{ Simpsoneven } \\
\hline & Mean & SD & Mean & SD & Mean & SD & Mean & SD & Mean & SD & Mean & SD \\
\hline$\overline{\mathrm{PE}}$ & $1350.56^{*}$ & 200.94 & $1421.26^{*}$ & 215.43 & $7.83^{*}$ & 0.49 & 1.02 & 0.01 & 0.98 & 0.00 & 0.05 & 0.02 \\
\hline$E$ & $1143.95^{*}$ & 215.08 & $1206.85^{*}$ & 234.63 & $7.44^{*}$ & 0.53 & 1.03 & 0.02 & 0.98 & 0.00 & 0.05 & 0.02 \\
\hline
\end{tabular}

$S D$ Standard Deviation. Asterisk indicates significant difference ( $p<0.05$, Student's t-test). $P E$ pre-eradicated group, $E$ eradicated group

"Chao 1, "ACE, and ${ }^{*}$ Shannon index between PE and E was statistically significant different $(p<0.05)$ 


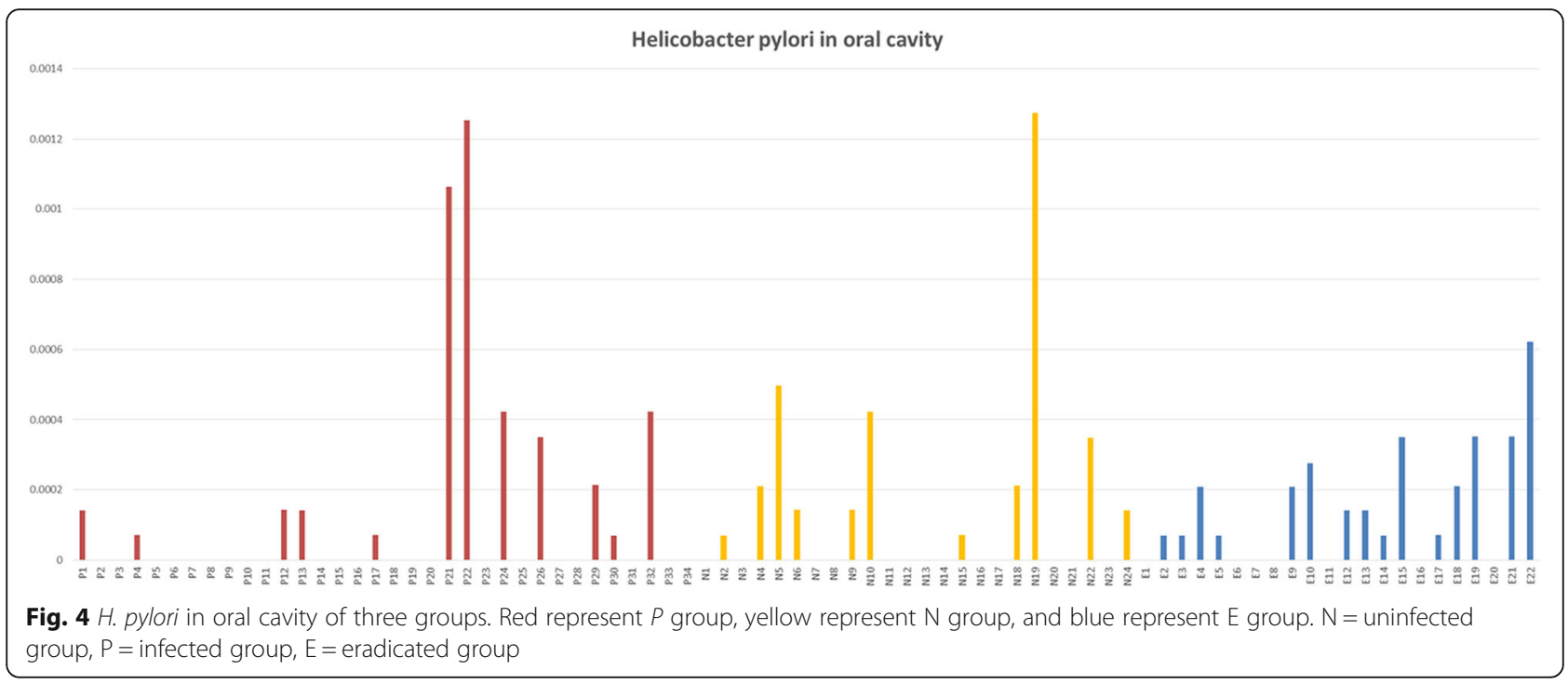

pylori in the oral cavity, respectively, $(p=0.054)$. The $H$. pylori signature was present in the saliva of subjects with negative ${ }^{13} \mathrm{C}$-UBT, RUT, and in $H$. pylori infected individuals after successful $H$. pylori eradication. We've also compared the prevalence of $H$. pylori both before and after H. pylori eradication. Pre-eradiation 7 subjects has positive saliva and 15 were negative. After successful eradication, $H$. pylori was still detected from saliva in 6 of the 7 positive subjects. Interestingly, 9 in 15 subjects who were $H$. pylori negative before eradication had it detected post $H$. pylori eradication whereas 6 remained free of $H$. pylori after eradication.

\section{Discussion}

In this study, using the technology of high-throughput sequencing, we found $H$. pylori exit in the oral cavity of infected (12/34), uninfected (11/24) and successful eradicated $(15 / 22)$ subjects, composing $0.0139 \%$ of the total sequences.

A few studies have shown that $H$. pylori can be spread by oral-oral (or fecal-oral) way [2]. Feeding, kissing, and tableware shared play the important role in the transmission. Previous studies have detected the presence of $H$. pylori in saliva [3]. Bacteria in plaque adhere to the gums, which are relatively fixed. It is more reasonable to study the saliva instead of dental plaque in this situation. A comprehensive and thorough investigation of the bacterial diversity of salivary microbiota is essential for understanding the how or whether $H$. pylori infection alters the salivary microbiota. The technology of high-throughput sequencing has provided new cognizance of the structures and compositions of microbiota communities.

By comparing the alpha diversity indexes we found that the bacterial diversity in saliva was similar among the $H$. pylori uninfected and $H$. pylori infected people. Our study is consistent with the notion that $H$. pylori in the stomach has little or no effect on the bacterial diversity of the oral cavity [20]. The Shannon diversity index, ACE richness index, and Chao 1 richness estimator all declined after eradication of $H$. pylori compared to the baseline samples $(p<0.05)$, which was consistent with the prior studies that use of PPI and antibiotics may affect the oral microbiome [21,22].

According to the beta diversity analysis based on the unweighted UniFrac distances, the community structures of saliva microbiota were different in H. pylori uninfected and infected individuals, which was contrary to the results of Schulz's study [20]. Samples from the $H$. pylori infected subjects tended to cluster together, while the microbiota in the uninfected subjects appeared to be more variable suggesting that gastric $H$. pylori infection may affect oral bacterial components. Clear segregations by the PCoA and NMDS analysis among individuals before and after $H$. pylori eradication therapy demonstrated that successful eradication or eradication therapy changed the oral bacterial components to some extent.

In addition to the presence of different bacterial members, the abundance of some bacteria also differed significantly among groups. We clearly observed that some bacteria in the saliva of $H$. pylori infected individuals showed a significantly reduced abundance, among which Aggregatibacter, Klebsiella, Fusobacterium, and Parvimonas species were pathogenic bacteria, causing infective endocarditis, liver abscess, pneumonia, meningitis, systemic infections, et al. [23-25]. However, the abundance of other bacteria significantly increased in saliva of $H$. pylori infected individuals, most of which were oral microbiota composition, including Sphingomonas, Ochrobactrum, Afipia, Leptotrichia, Oribacterium, and Moryell, except Acinetobacter species causing infectious diseases like pneumonia and urinary tract infections 
[26], and Leptotrichia species, a potential cariogenic bacteria [27]. While in Schulz's study, no significant difference in oral communities between $H$. pylori infected and uninfected individuals was detected at genus level [21], this may be due to the different target sequencing region of $16 \mathrm{~s}$ rDNA, sample size, or geographic location. Interestingly, most $H$. pylori-enriched genera increased after the eradication, including Ralstonia, Leptotrichia, Sphingomonas, Leptothrix, Oribacterium, and Acinetobacter. The exception was Ochrobactrum. However, genera low expressed in $H$. pylori infected saliva experienced a further decline after $H$. pylori eradication therapy, including Alloprevotella, Aggregatibacter, Leptotrichlaceae_G_1_, Parvimonas, and Fusobacterium. Our study suggests that $H$. pylori infection may change the salivary microbiota, eradication therapy would further change the bacteria composition of saliva microbiota. Although the clinical significance of these alterations was not known, $H$. pylori unexpectedly and clearly altered the oral microbiota composition.

In our study, we found that $H$. pylori infection didn't change the abundance and diversity, but changed the composition structure of salivary microbiome. $\mathrm{H}$. pylori is colonized in the stomach, and there is a certain distance from stomach to the oral cavity. Even though $H$. pylori can cause a large change in the abundance of the microbiota in the stomach, it is difficult to affect the abundance of the salivary microbial flora. Previous studies have reported acid inhibition in upper gastric tract may have an effect on the oral microbiome leading to alterations in the microbiota [28]. In addition, changes in gastric $\mathrm{pH}$ could also lead to an alteration in the $\mathrm{pH}$ of oral cavity [29]. We proposed that $H$. pylori likely changed the community and structure of oral microbiota through changes in the acidic environment in stomach by generating large amount of urease, an enzyme which decomposes urea into ammonia and carbon dioxide and transiently reduce the acidic environment in the stomach [30], leading to the further changes in $\mathrm{pH}$ of oral cavity. The use of PPIs during the eradication therapy would further inhibit the $\mathrm{pH}$ in stomach, leading to further alteration in saliva microbiota, which can partially explain why genera enriched in $H$. pylori infected individuals would further increase and genera low expressed in H. pylori infected individuals would decline after successful eradication. Although the precise mechanism has yet to be clarified, to our knowledge this is the first study to clearly show oral microbiota alterations as a result of H. pylori infection in a cohort of subjects. Additional studies like metagenomics sequencing or metabolomics to investigate these possible causal relationships would likely provide interesting findings.

Using amplicon sequencing of $16 \mathrm{~S}$ rDNA V3-V4 hypervariable regions we detected $H$. pylori in the oral cavity of almost half of the subjects regardless of whether they had gastric infection with H. pylori. Subjects who did not have $H$. pylori in the oral cavity before eradication surprisingly had $H$. pylori detected in saliva samples after $H$. pylori eradication therapy. Clearly, using these techniques the prevalence of $\mathrm{H}$. pylori in oral cavity is not clearly associated with colonization status in the stomach which is not consistent with the notion that the oral cavity represents a secondary site for $H$. pylori colonization [31]. The gastric and oral mucosa differ markedly. For example, of the two only the gastric mucosa expresses Lewis ${ }^{\mathrm{b}}$ antigen, an $\mathrm{ABO}$ blood group antigen, which enables adherence of H. pylori to the epithelial surfaces. It has been proposed that $H$. pylori is a passerby in oral cavity, rather than a colonizer and it may be also be included in the material in gastroesophageal reflux. The natural history of $H$. pylori infection has been that after $H$. pylori eradication from the stomach, gastric reinfection is rare and when it occurs early it can often be shown to be recrudescence (the same genotype) whereas later reinfections are most often reinfection with a different genotype [32]. The hypothesis that $H$. pylori was a common passerby rather than a colonizer would partly explain why recurrences are most common in areas with poor sanitation and a high prevalence of $H$. pylori and rare in developed countries whose frequency of $H$. pylori infection had become much lower than that of poor regions.

\section{Strengths and limitations}

Our study showed that $H$. pylori infection and the eradication treatment resulted in alterations of oral microbiota. However, there were limitations to our study. The technique of high-throughput sequencing we used in our study could not detect $H$. pylori-specific virulence factors such as VacA, CagA, OipA, etc. or full sequence [33]. Although the samples of the first set of crosssectional analysis were collected at different times in $\mathrm{Au}-$ gust 2018, the collection interval between the first and the last collection were equal. All the samples underwent DNA extraction and sequencing analysis at the same time to make our result were comparable. One issue with the interpretation is that there was no control sample of $H$. pylori uninfected individuals receiving the same antimicrobial therapy which precluded determination about whether the presence of $H$. pylori, the antimicrobial therapy, or both were dominant factors in changing the within-individual diversity of the oral cavity.

\section{Conclusions}

Our study using high-throughput sequencing showed that H. pylori is present commonly in the oral cavity with no clear relation to $H$. pylori infection of the stomach. Both $H$. pylori infection and eradication therapy caused alterations in community and structure of the oral microbiota. 


\section{Supplementary information}

Supplementary information accompanies this paper at https://doi.org/10. 1186/s12903-020-01070-1.

Additional file 1: Table S1. Clinical parameters of the 58 subjects. $N=$ uninfected group, $\mathrm{P}=$ infected group, $\mathrm{PE}=$ pre-eradicated group, $\mathrm{E}=$ eradicated group.

Additional file 2: Table S2. Barcodes of all samples subjected to multiplex sequencing. $\mathrm{N}=$ uninfected group, $\mathrm{P}=$ infected group, $\mathrm{E}=$ eradicated group.

Additional file 3: Table S3. Sequences and modified OTUs of all saliva samples from sequencing at $97 \%$ identity. $N=$ uninfected group, $P=$ infected group, $\mathrm{E}=$ eradicated group.

Additional file 4: Figure S1. Length distribution of sequences determined by Illumina MiSeq sequencing.

Additional file 5: Table S4. Modified OTU table at 97\% identity. $N=$ uninfected group, $\mathrm{P}=$ infected group, $\mathrm{E}=$ eradicated group.

Additional file 6: Figure S2. A classification tree showing bacterial abundance by MEGAN. The taxonomy compositions and abundances were visualized by MEGAN (version 6.6.7). The larger the area of the colored pie chart, the greater the bacterial abundance. Different colors represent different groups, and the larger the colored sectorial area at a branch, the more the corresponding group contributed to the bacterial abundance. $\mathrm{N}=$ uninfected group, $\mathrm{P}=$ infected group, $\mathrm{E}=$ eradicated group.

Additional file 7: Figure S3. Alpha diversity (observed species number) among groups. A. $\mathrm{N}$ group and $\mathrm{P}$ group showed similar alpha diversity ( $p>0.05$ ). $N=$ uninfected group, $P=$ infected group. B. The observed species in $\mathrm{E}$ group were significantly lower than that of PE group and $\mathrm{N}$ group ( $p<0.01)$; One asterisk indicates significant differences $(p<0.05$, Student's t-test), two asterisk indicates $p<0.01$, three asterisk indicates $p<0.001 . \mathrm{N}=$ uninfected group, $\mathrm{PE}=$ pre-eradicated group, $\mathrm{E}=$ eradicated group.

Additional file 8: Figure S4. Rank abundance curves for all OTUs. N = uninfected group, $\mathrm{P}=$ infected group, $\mathrm{E}=$ eradicated group.

Additional file 9: Figure S5. Nonmetric Multidimensional Scaling (NMDS) based on unweighted UniFrac distances at the OUT level at $97 \%$ identity. Each sample is represented by a dot. A. The samples formed well-separated clusters corresponding to the two groups, suggesting that the bacterial structures in $\mathrm{N}$ group and $\mathrm{P}$ group were different. $\mathrm{N}=$ uninfected group, $\mathrm{P}=$ infected group. Red squares represent the $\mathrm{N}$ samples. Blue triangles represent the $\mathrm{P}$ samples. B. Blue triangles represent the $\mathrm{N}$ samples. Red circles represent the $\mathrm{E}$ samples. The samples formed wellseparated clusters corresponding to the three groups, suggesting that the bacterial structures in E group, PE group, and $\mathrm{N}$ group were different. $\mathrm{N}=$ uninfected group, $\mathrm{PE}=$ pre-eradicated group, $\mathrm{E}=$ eradicated group.

Additional file 10: Figure S6. Comparison of microbial variations at the genus level, using the LEfSe online tool. A. Histogram of the LDA scores for differentially abundant features among groups. The threshold on the logarithmic LDA score for discriminative features was set to 2.0. $\mathrm{N}=$ uninfected group, $\mathrm{PE}=$ pre-eradicated group, $\mathrm{E}=$ eradicated group. $\mathbf{B}$. Cladogram for taxonomic representation of significantly differences among groups. Differences are represented in the color of the most abundant taxa (red indicating $\mathrm{N}$ group, blue indicating PE group, green indicating $E$ group, and white indicating non-significant). $N=$ uninfected group, $\mathrm{PE}=$ pre-eradicated group, $\mathrm{E}=$ eradicated group.

\section{Abbreviations}

H. pylori: Helicobacter pylori; OTUs: Operational taxonomic units; PCR: Polymerase chain reaction; QIIME: Quantitative Insights Into Microbial Ecology; HOMD: Human Oral Microbiome Database; ACE: Abundance-based Coverage Estimator; PCoA: Principal coordinate analysis; NMDS: Nonmetric Multidimensional Scaling; LEfSe: Linear discriminant analysis effect size; ${ }^{13} \mathrm{C}$ UBT: ${ }^{13} \mathrm{C}$ urea breath test; RUT: Rapid urease test; PPI: Proton pump inhibitor; SD: Standard Deviation; N: Uninfected group; P: Infected group; E: Eradicated group; PE: Pre-eradicated group

\section{Acknowledgments}

We acknowledged Shanghai Personal Biotechnology Co., Ltd. for their kind help in Illumina MiSeq Sequencing and bioinformatics analysis. This manuscript has been released as a Pre-Print at BioRxiv [34]

\section{Authors' contributions}

YJJ performed the literature review and experimental analysis, $X L$ built the updated version of the datasets, and $\mathrm{HL}$ supervised the work. YJJ and XL drafted the manuscript. All the authors have read and approved the final manuscript.

\section{Funding}

This study was funded by National Natural Science Foundation of China (grant number 81170355 and 81370592) and Clinical Research Center, Shanghai Jiao Tong University School of Medicine, which played no role in the design of the study and collection, analysis, and interpretation of data and in writing the manuscript.

\section{Availability of data and materials}

The datasets analysed during the current study are available in the NCBI Sequence Read Archive at https://www.ncbi.nlm.nih.gov/ under accession number SRP167714. The study protocol and statistical analysis plan are available online from https://clinicaltrials.gov/ct2/show/ NCT03730766.

\section{Ethics approval and consent to participate}

The present study protocol was reviewed and approved by the Ethics Committee of the Renji Hospital of Shanghai Jiao Tong University (ethics application no. 2016[63 k]). Before beginning work, we orally explained our study objectives and procedures to all participants, and obtained their permission to have their saliva specimens involved in our study. Meanwhile, written informed consents were signed by each participant. Each head of the selected households was responsible for giving them to us.

\section{Consent for publication}

Not applicable.

\section{Competing interests}

The authors declare that they have no competing interests.

Received: 26 November 2019 Accepted: 10 March 2020

Published online: 20 March 2020

\section{References}

1. Malfertheiner P, Megraud F, O'Morain CA, Gisbert JP, Kuipers EJ, Axon AT, et al. Management of Helicobacter pylori infection-the Maastricht V/ Florence consensus report. Gut. 2017:66(1):6-30.

2. Bui D, Brown HE, Harris RB, Oren E. Serologic evidence for fecal-Oral transmission of helicobacter pylori. Am J Trop Med Hyg. 2016;94(1):82-8.

3. Yee JK. Helicobacter pylori colonization of the oral cavity: a milestone discovery. World J Gastroenterol. 2016;22(2):641-8.

4. Moodley A, Wood NH, Shangase SL. The relationship between periodontitis and diabetes: a brief review. SADJ. 2013;68(6):260, 262-4.

5. Zhou J, Jiang N, Wang Z, Li L, Zhang J, Ma R, et al. Influences of pH and Iron Concentration on the Salivary Microbiome in Individual Humans with and without Caries. Appl Environ Microbiol. 2017;83(4).

6. Isola G, Polizzi A, Santonocito S, Alibrandi A, Ferlito S. Expression of salivary and serum malondialdehyde and lipid profile of patients with periodontitis and coronary heart disease. Int J Mol Sci. 2019;20(23):E6061.

7. Isola G, Matarese M, Ramaglia L, lorio-Siciliano V, Cordasco G, Matarese G. Efficacy of a drug composed of herbal extracts on postoperative discomfort after surgical removal of impacted mandibular third molar: a randomized, triple-blind, controlled clinical trial. Clin Oral Investig. 2019;23(5):2443-53.

8. Eick S, Nydegger J, Bürgin W, Salvi GE, Sculean A, Ramseier C. Microbiological analysis and the outcomes of periodontal treatment with or without adjunctive systemic antibiotics-a retrospective study. Clin Oral Investig. 2018:22(9):3031-41.

9. Ciric L, Pratten J, Wilson M, Spratt D. Development of a novel multi-triplex qPCR method for the assessment of bacterial community structure in oral populations. Environ Microbiol Rep. 2010;2(6):770-4. 
10. Chen $\mathrm{H}$, Jiang W. Application of high-throughput sequencing in understanding human oral microbiome related with health and disease. Front Microbiol. 2014;5:508.

11. Kuczynski J, Stombaugh J, Walters WA, González A, Caporaso JG, Knight R. Using QIIME to analyze 16S rRNA gene sequences from microbial communities. Curr Protoc Bioinformatics 2011. https://doi.org/10.1002/ 0471250953.bi1007s36.

12. Edgar RC. Search and clustering orders of magnitude faster than BLAST. Bioinformatics (Oxford, England). 2010. https://doi.org/10.1093/ bioinformatics/btq461.

13. Kuska B. Scientists Launch First Comprehensive Database of Human Oral Microbiome. 2008. http://www.nider.nih.gov/Research/ResearchResults/ NewsReleases/ArchivedNewsReleases/NewsReleases2008/OralMicrobiome. htm.

14. Bokulich NA, Subramanian S, Faith JJ, Gevers D, Gordon Jl, Knight R, et al. Quality-filtering vastly improves diversity estimates from Illumina amplicon sequencing. Nat Methods 2013. https://doi.org/10.1038/nmeth.2276.

15. Paul D, Kumbhare SV, Mhatre SS, Chowdhury SP, Shetty SA, Marathe NP, et al. Exploration of microbial diversity and community structure of Lonar Lake: the only Hypersaline meteorite crater Lake within basalt rock. Front Microbiol. 2015;6:1553.

16. Ramette A. Multivariate analyses in microbial ecology. FEMS Microbiol Ecol. 2007:62(2):142-60.

17. David IW, Stephen TW, Yi W. Distance-based multivariate analyses confound location and dispersion effects. Methods Ecol Evol. 2012;3(1):89-101.

18. Asnicar F, Weingart G, Tickle TL, Huttenhower C, Segata N. Compact graphical representation of phylogenetic data and metadata with GraPhIAn. PeerJ. 2015:3:e1029.

19. Segata N, Izard J, Waldron L, Gevers D, Miropolsky L, Garrett WS, et al. Metagenomic biomarker discovery and explanation. Genome Biol. 2011; 12(6):R60.

20. Schulz C, Schütte K, Koch N, Vilchez-Vargas R, Wos-Oxley ML, Oxley APA, et al. The active bacterial assemblages of the upper $\mathrm{Gl}$ tract in individuals with and without infection. Gut. 2018. https://doi.org/10.1136/gutjnl-2016312904

21. Abeles SR, Ly M, Santiago-Rodriguez TM, Pride DT. Effects of long term antibiotic therapy on human Oral and fecal Viromes. PLoS One. 2015;10(8): e0134941.

22. Mishiro T, Oka K, Kuroki Y, Takahashi M, Tatsumi K, Saitoh T, et al. Oral microbiome alterations of healthy volunteers with proton pump inhibitor. J Gastroenterol Hepatol 2018. https://doi.org/10.1111/jgh.14040.

23. Nørskov-Lauritsen N. Classification, identification, and clinical significance of Haemophilus and Aggregatibacter species with host specificity for humans. Clin Microbiol Rev 2014. https://doi.org/10.1128/cmr.00103-13.

24. Shon AS, Bajwa RP, Russo TA. Hypervirulent (hypermucoviscous) Klebsiella pneumoniae: a new and dangerous breed. Virulence. 2013. https://doi.org/ 10.4161/viru.22718.

25. Chaucer B, Smith N, Beatty D, Yadav M. Multiple hepatic abscess from : an emerging gastrointestinal microbe. ACG Case Rep J. 2018;5:e70.

26. Wong D, Nielsen TB, Bonomo RA, Pantapalangkoor P, Luna B, Spellberg B. Clinical and pathophysiological overview of Acinetobacter infections: a century of challenges. Clin Microbiol Rev. 2017;30(1):409-47.

27. Belstrøm D, Sembler-Møller ML, Grande MA, Kirkby N, Cotton SL, Paster BJ, et al. Impact of Oral hygiene discontinuation on Supragingival and salivary microbiomes. JDR Clin Trans Res. 2018;3(1):57-64.

28. Johnstone J, Nerenberg K, Loeb M. Meta-analysis: proton pump inhibitor use and the risk of community-acquired pneumonia. Aliment Pharmacol Ther. 2010;31(11):1165-77.

29. Jin JS, Touyama M, Yamada S, Yamazaki T, Benno Y. Alteration of a human intestinal microbiota under extreme life environment in the Antarctica. Biol Pharm Bull. 2014;37(12):1899-906

30. Sugano K, Tack J, Kuipers EJ, Graham DY, El-Omar EM, Miura S, et al. Kyoto global consensus report on helicobacter pylori gastritis. Gut. 2015. https:// doi.org/https://doi.org/10.1136/gutjnl-2015-309252.

31. Zou QH, Li RQ. Helicobacter pylori in the oral cavity and gastric mucosa: a meta-analysis. J Oral Pathol Med 2011. https://doi.org/https://doi.org/10. 1111/j.1600-0714.2011.01006.x.

32. Kim SY, Hyun JJ, Jung SW, Koo JS, Yim HJ, Lee SW. Helicobacter pylori recurrence after first- and second-line eradication therapy in Korea: the problem of recrudescence or reinfection. Helicobacter. 2014. https://doi.org/ https://doi.org/10.1111/hel.12117.
33. Sugimoto M, Wu JY, Abudayyeh S, Hoffman J, Brahem H, Al-Khatib K, et al. Unreliability of results of PCR detection of helicobacter pylori in clinical or environmental samples. J Clin Microbiol 2009. https://doi.org/https://doi. org/10.1128/jcm.01563-08

34. Ji YJ, Liang X, Lu H. The Salivary Microbiome: Analysis of by Pyrosequencing and the Relationship with Helicobacter pylori Infection. bioRxiv. 2018. https://doi.org/. https://doi.org/10.1101/505115.

\section{Publisher's Note}

Springer Nature remains neutral with regard to jurisdictional claims in published maps and institutional affiliations.
Ready to submit your research? Choose BMC and benefit from:

- fast, convenient online submission

- thorough peer review by experienced researchers in your field

- rapid publication on acceptance

- support for research data, including large and complex data types

- gold Open Access which fosters wider collaboration and increased citations

- maximum visibility for your research: over $100 \mathrm{M}$ website views per year

At BMC, research is always in progress.

Learn more biomedcentral.com/submissions 